\title{
Avaliação Comparativa entre Valvoplastia Percutânea e Comissurotomia a Céu Aberto na Estenose Mitral
}

\author{
Luiz Francisco Cardoso, Miguel Antonio Neves Rati, Pablo Maria Alberto Pomerantzeff, Caio Cesar \\ Jorge Medeiros, Flávio Tarasoutchi, Eduardo Giusti Rossi, Walkiria Samuel Á vila, Max Grinberg
}

São Paulo, SP

\begin{abstract}
Objetivo - Comparar resultados imediatos e após 12 meses de seguimento entre valvoplastia por balão e comissurotomia na estenose mitral.

Métodos - Oitenta e oito portadores de estenose mitral sintomáticos com anatomia favorável foram randomizados nos dois grupos. Todos os pacientes submeteram-se a avaliação clínica e Doppler ecocardiográfica antes, imediatamente após e 12 meses depois do procedimento.

Resultados-Gradiente médio mitral $(\mathrm{mmHg})$ diminuiu

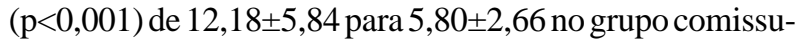
rotomia (GC) e de 11,66 $\pm 6,13$ para 4,98 $\pm 2,40$ ( $\mathrm{p}<0,001)$ no grupo valvuloplastia $(\mathrm{GV})$. Área valvar mitral $\left(\mathrm{cm}^{2}\right)$ aumentou de $0,98 \pm 0,21$ para $2,52 \pm 0,46$ no GC e de $1,05 \pm 0,25$ para $2,18 \pm 0,40$ no $\mathrm{GV}$ ( $\mathrm{p}<0,001)$. Em ambos os grupos ocorreu uma diminuição discreta na área valvar mitral ao final do acompanhamento. Não ocorreram óbitos. Um paciente do GV apresentou insuficiência mitral significativa e necessitou tratamento cirúrgico. Aos 12 meses de acompanhamento todos os pacientes do GC e 97,7\% dos pacientes do GV estavam em classe funcional I ou II (NYHA).

Conclusão - Os procedimentos foram seguros e mostraram melhora semelhante no gradiente mitral e classe funcional. A área valvar mitral aumentou expressivamente mais no GC, e apresentou, também, uma redução mais significativa após 12 meses. A maioria dos pacientes não alterou a classe funcional no acompanhamento.
\end{abstract}

Palavras-chave: estenose mitral, valvuloplastia por balão, comissurotomia

\section{Comparison Between Percutaneous Balloon Valvuloplasty and Open Commissurotomy for Mitral Stenosis}

Purpose - To compare immediate and late (12 months) follow up of clinical and Doppler echocardiographic results between percutaneous mitral balloon valvuloplasty and open commissurotomy in a prospective and randomized trial.

Methods - Eighty eight symptomatic patients with severe mitral stenosis and favorable anatomy were randomized in a prospective trial comparing the two procedures. All patients were submitted to clinical and Doppler echocardiographic evaluation before the procedures and immediate and twelve months thereafter.

Results - Mean mitral gradient ( $\mathrm{mmHg}$ ) decreased from $12.2 \pm 5.8$ to $5.80 \pm 2.7(\mathrm{p}<0.001)$ in commissurotomy group (CG) and from $11.7 \pm 6.1$ to $5.0 \pm 2.4(\mathrm{p}<0.001)$ in the balloon valvuloplasty group (VG). Mitral valve area $\left(\mathrm{cm}^{2}\right)$ increased from $0.98 \pm 0.21$ to $2.52 \pm 0.46$ in CG and from $1.05 \pm 0.25$ to $2.18 \pm 0.40$ in $\mathrm{VG}(\mathrm{p}<0.001)$. In both groups there was a slight decrease in mitral valve area at 12 month follow-up. There was no death in either group. One patient in the VG had moderate mitral regurgitation and underwent surgery. At the 12 month follow-up, all patients in CG and $97.7 \%$ of patients in VG were in New York Heart Association functional class I or II.

Conclusion - Both procedures were safe and showed similar immediate improvement in mitral gradient and functional class. Mitral valve area had a greater increase immediately after commissurotomy, however there was a significantly greater reduction in the CG after 12 months of followup, when compared to balloon valvuloplasty. In both groups, mitral gradient remained reduced and most patients did not change functional class during the follow-up.

Key-words: mitral valve stenosis, balloon valvuloplasty, commissurotomy

Arq Bras Cardiol, volume 70 (nº 6), 415-421, 1998

Instituto do Coração do Hospital das Clínicas - FMUSP

Correspondência: Luiz Francisco Cardoso - Incor - Divisão de Clínica - Av. Dr Enéas C. Aguiar, 44 - 05403-000 - São Paulo, SP

Recebido para publicação em 29/1/98

Aceito em 11/3/98
A estenose mitral (EM), cardiopatia reumática altamente prevalente em nosso país ${ }^{1}$, persiste, despertando grande interesse no aprimoramento de técnicas para sua correção.

A partir de 1984, a valvoplastia mitral por cateter balão (VMCB) foi introduzida por Inoue e $\mathrm{col}^{2} \mathrm{e}$, de pronto, incor- 
porada à prática diária, tornando-se alternativa à clássica e eficaz intervenção cirúrgica ${ }^{3-5}$.

Todavia, os métodos não são superponíveis, sendo a VMCB passível de ser influenciada pelas peculiaridades morfológicas de cada caso.

Este estudo compara, ao longo de um ano de seguimento, estes dois métodos terapêuticos.

\section{Métodos}

Foram selecionados 88 portadores de EM com as seguintes características: a) classe funcional (CF) II,III e IV (NYHA) ${ }^{6}$; b) escore Dopplerecocardiográfico ${ }^{7} \leq 9$; c) idade $<60$ anos; d) ausência de insuficiência mitral (IM), além de grau discreto; e) ausência de outra cardiopatia com indicação cirúrgica; f) ausência de história de embolia; g) ausência de trombo intracavitário ao ecocardiograma. Foram constituídos dois grupos, através de randomização (tab. I): a) grupo comissurotomia (GC) - 44 casos; b) grupo valvoplastia (GV)- 44 casos.

Os procedimentos foram efetuados pelo mesmo cirurgião e hemodinamicista. A técnica do duplo balão ${ }^{8}$ (Boston Scientific, Mass) foi empregada em quatro (9\%) pacientes. Sete $(16,0 \%)$ pacientes foram submetidos à VMCB com balão bifoil (Schneider Medintag) e os restantes $33(75,0 \%)$ à VMCB pela técnica de Inoue ${ }^{2}$.

A avaliação clínico-laboratorial constou de: a) anamnese e exame físico completos no PRÉ(imediatamente antes do procedimento), POI (imediatamente antes da alta hospitalar) e PO12M (após 12 meses de acompanhamento), observando-se os dados da $\mathrm{CF}$, complicações cardíacas e eventos extracardíacos; b) eletrocardiograma nas 12 derivações habituais no PRÉ e PO12M, considerando-se apenas o ritmo cardíaco, sinusal, juncional ou fibrilação atrial; c) ecocardiograma no PRÉ, POI e PO12M, segundo critérios da Associação Americana de Ecocardiografia ${ }^{9}$. Foram analisados: a) gradiente transvalvar mitral médio $(\mathrm{G}) \mathrm{em} \mathrm{mmHg}$; b) área valvar mitral (AVM) $\mathrm{em} \mathrm{cm}^{2}$, pelo pressure half time; c) grau de IM, classificado em ausente, discreta (+), moderada (2+), importante (3+), através do Doppler pulsado e con- tínuo e pelo mapeamento de fluxo a cores; d) escore ecocardiográfico segundo os critérios de Wilkins e col ${ }^{7}$.

A CF, ritmo cardíaco, escore ecocardiográfico, $\mathrm{G} \mathrm{e}$ AVM ao ecocardiograma no PRÉ estão expostos na tabela I. Não houve diferença significativa entre os grupos em relação a estas variáveis no pré-procedimento.

A análise da eficácia dos procedimentos obedeceu à aplicação de critério consagrado de sucesso ${ }^{10}$, ou seja, AVM no POI calculada pelo ecocardiograma $\geq 1,5 \mathrm{~cm}^{2}$ ou pelo menos $50 \%$ superior à do pré-procedimento.

A análise descritiva dos dados consistiu do cálculo das médias e desvio-padrão para as variáveis quantitativas e do cálculo de frequiências absolutas e relativas para as variáveis qualitativas. Para as variáveis quantitativas, compararam-se as médias através do teste t de Student para amostras independentes. Para as variáveis qualitativas, a hipótese de homogeneidade de proporções entre os dois grupos foi avaliada, utilizando-se o teste de qui-quadrado ou o teste exato de Fisher. Comparação entre os grupos no PRÉ, POI e PO12M para as variáveis do ecocardiograma utilizou análise de variância com medidas repetidas.

\section{Resultados}

A evolução da CF no PRÉ, POI e PO12M em ambos os grupos está representada na figura 1. Houve melhora expressiva da $\mathrm{CF}$ em ambos os grupos. Todos os casos do GC evoluíram para CFI/II no POI e a mantiveram no PO12M [em dois $(4,5 \%)$ casos houve perda de seguimento]. Quarenta e três $(97,7 \%)$ casos do GV evoluíram para CF I/II no POI e a mantiveram no PO12M. Um (2,3\%) caso do GV permaneceu em CF III no POI (submetido à correção de dupla disfunção mitral pós-VMCB porimplante debioprótese mitral após 42 dias).

O comportamento da média do $\mathrm{G}$ médio não mostrou diferença significante $\left(\mathrm{H}_{01}: \mathrm{p}=0,1689 ; \mathrm{H}_{02}: \mathrm{p}=0,6932\right)$ entre os grupos ao longo do estudo (fig. 2 e tab. II). Os valores médios no POI mostraram-se diferentes em relação ao PRÉ $(\mathrm{p}<0,0001)$ e semelhantes em relação aos do PO12M $(\mathrm{p}=0,1004)$.

Houve diferença significante $\left(\mathrm{H}_{01}: \mathrm{p}=0,0002\right)$ dos valo-

\begin{tabular}{|c|c|c|c|}
\hline Variável & $\begin{array}{c}\text { Comissurotomia } \\
(\mathrm{n}=44)\end{array}$ & $\begin{array}{l}\text { Valvoplastia } \\
\qquad(\mathrm{n}=44)\end{array}$ & $P$ \\
\hline Idade (anos) & $31,6 \pm 8,5(20-54)$ & $32,2 \pm 9,5(15-54)$ & 0,741 \\
\hline Sexo(fem/masc) & $37 / 7$ & $40 / 4$ & 0,334 \\
\hline Classe funcional (NYHA) & & & 0,713 \\
\hline II & 3 & 5 & \\
\hline $\mathrm{III} / \mathrm{IV}$ & 41 & 39 & \\
\hline Ritmo cardíaco & & & 0,196 \\
\hline sinusal & 36 & 41 & \\
\hline Fibrilação atrial/juncional & 8 & 3 & \\
\hline Escore ecocardiográfico ${ }^{7}$ & & & 0,727 \\
\hline $4 / 5$ & 5 & 6 & \\
\hline $6 / 7 / 8$ & 37 & 34 & \\
\hline 9 & 2 & 4 & \\
\hline Gradiente mitral (mmHg) & $12,2 \pm 5,8$ & $11,7 \pm 6,1$ & 0,2987 \\
\hline Área valvar mitral $\left(\mathrm{cm}^{2}\right)$ & $0,98 \pm 0,21$ & $1,05 \pm 0,25$ & 0,007 \\
\hline
\end{tabular}




\begin{tabular}{|c|c|c|c|c|c|c|}
\hline & \multicolumn{2}{|c|}{ Pré } & \multicolumn{2}{|c|}{ Imediato } & \multicolumn{2}{|c|}{12 Meses } \\
\hline & $\mathrm{CM}$ & VMCB & $\mathrm{CM}$ & VMCB & $\mathrm{CM}$ & VMCB \\
\hline AVM & $0,98 \pm 0,21$ & $1,05 \pm 0,25$ & $2,52 \pm 0,46$ & $2,18 \pm 0,40$ & $2,13 \pm 0,32$ & $2,02 \pm 0,42$ \\
\hline $\mathrm{G}$ & $12,2 \pm 5,8$ & $11,7 \pm 6,1$ & $5,8 \pm 2,7$ & $5,0 \pm 2,4$ & $5,2 \pm 1,9$ & $5,3 \pm 3,0$ \\
\hline
\end{tabular}

\begin{tabular}{|c|c|c|c|}
\hline PRÉ & PO12M & $\mathrm{N}$ & $\%$ \\
\hline \multicolumn{4}{|c|}{ Comissurotomia } \\
\hline Sinusal & Sinusal & 36 & 81,8 \\
\hline Fibrilação atrial & Fibrilação atrial & 4 & 9,1 \\
\hline Juncional & Sinusal & 4 & 9,1 \\
\hline \multicolumn{4}{|c|}{ Valvoplastia } \\
\hline Sinusal & Sinusal & 41 & 93,2 \\
\hline Fibrilação atrial & Fibrilação atrial & 3 & 6,8 \\
\hline
\end{tabular}

\begin{tabular}{|c|c|c|}
\hline \multicolumn{3}{|c|}{$\begin{array}{l}\text { Tabela IV- Complicações cardíacas não valvares no POI em } \\
\text { ambos os grupos. }\end{array}$} \\
\hline $\mathrm{N}$ & $\%$ & Tipo \\
\hline \multicolumn{3}{|r|}{ Comissurotomia } \\
\hline 6 & 13,6 & Fibrilação atrial com pericardite \\
\hline 1 & 2,3 & Bradicardia juncional com marca passo provisório \\
\hline 1 & 2,3 & Lesão de átrio direito \\
\hline \multicolumn{3}{|r|}{ Valvoplastia } \\
\hline 2 & 4,6 & Tamponamento cardíaco \\
\hline 1 & 2,3 & Perfuração de átrio direito com derrame pericárdico \\
\hline
\end{tabular}

res médios da AVM no PRÉ, POI e PO12M entre GV e GC (fig. 3 e tab. II). Em ambos os grupos, observaram-se aumento do PRÉ para o POI ( $\mathrm{p}<0,0001)$ e, a seguir, diminuição no PO12M (p<0,0001).

A maioria $(90,9 \%)$ dos pacientes não sofreu modificação do ritmo cardíaco, mas quatro $(9,1 \%)$ do GC que apresentavam ritmo juncional no PRÉ evoluíram para sinusal no PO12M um manifestou fibrilação atrial paroxística (tab. III).

No POI do GC (fig. 4), IM moderada foi reconhecida em dois casos (ausentes no PRÉ), IM discreta em 14 (10 ausentes e 4 discretas no PRÉ). No POI do GV, o ecocardiograma (fig. 5) permitiu identificar IM moderada em quatro casos (3 ausentes e outra discreta no PRÉ) e IM discreta em 16 (9 ausentes e 7 com IM discreta no PRÉ).

No GC (fig. 4), dentre os 16 casos que apresentavam IM observada pelo ecocardiograma no POI, houve diminuição do grau em $9(56,2 \%)$ e aumento em um $(6,2 \%)$ caso (perda de seguimento em dois casos). IM apareceu em 12 $(42,8 \%)$ dos 28 casos em que estava ausente no POI (perda de seguimento em dois casos).

No GV (fig. 5), dentre 20 casos que apresentavam IM observada pelo ecocardiograma no POI, houve diminuição do grau em sete $(35,0 \%)$ e aumento em um $(5,0 \%)$ caso (perda de seguimento em um caso e implante de bioprótese em outro). IM apareceu em 9 (37,5\%) dos 24 casos em que estava ausente no POI (perda de seguimento em um caso).
Na tabela IV estão expostas as complicações cardíacas não valvares decorrentes dos procedimentos no POI. No PO12M, um (2,3\%) caso no GV desenvolveu bloqueio completo do ramo esquerdo e outro $(2,3 \%)$ comunicação interatrial, não documentada no POI.

No POI, três $(6,9 \%)$ casos do GC apresentaram anemia com necessidade de transfusão de sangue, 2 (4,6\%) pneumotórax e um $(2,3 \%)$ necessitou reoperação imediata por sangramento. Ocorreu gestação em uma (2,3\%) paciente do GV. Um (2,3\%) caso do GC apresentou acidente vascular cerebral isquêmico em presença de fibrilação atrial crônica e ausência do uso de anticoagulante.

\section{Discussão}

A história natural da EM reumática é caracterizada por longo período inicial assintomático. Admite-se que, após 16 anos do surto de febre reumática, $50 \%$ dos pacientes encontram-se assintomáticos, $35 \%$ em CFII, $8 \%$ em CFIII e $7 \%$ já tenham sido submetidos à cirurgia corretiva ${ }^{11}$.

$\mathrm{O}$ tratamento cirúrgico através de $\mathrm{CM}$ a céu aberto ou fechado tem mostrado ótimos resultados, com taxa de letalidade ${ }^{12}$ não superior a $3 \%$. Hickey e col ${ }^{15}$ observaram sobrevida de $99,7 \%, 99,0 \%, 95,0 \%, 87,0 \%$ e $59,0 \%$ em períodos de um mês, um, cinco, 10 e 20 anos de acompanhamento pós-operatório. Cerca de 78,0\% e 47,0\% desses pacientes ficaram livres de substituição da valva mitral no período de 10 e 20 anos, respectivamente. Todavia, SchmidtHabelmann e col ${ }^{5}$ verificaram necessidade de reoperação em $75 \%$ e $50 \%$ no periodo de 5 e 7,5 anos respectivamente, concluindo que a CM, não necessariamente, provoca modificações definitivas na progressão da doença valvar.

Os critérios de indicação de VMCB em portadores selecionados de $\mathrm{EM}^{10}$ superpõem-se em linhas gerais aos de indicação cirúrgica da EM, no entanto a aplicação da VMCB requer definição rigorosa de certos critérios morfofuncionais.

Nossa seleção de casos considerou apenas os candi- 


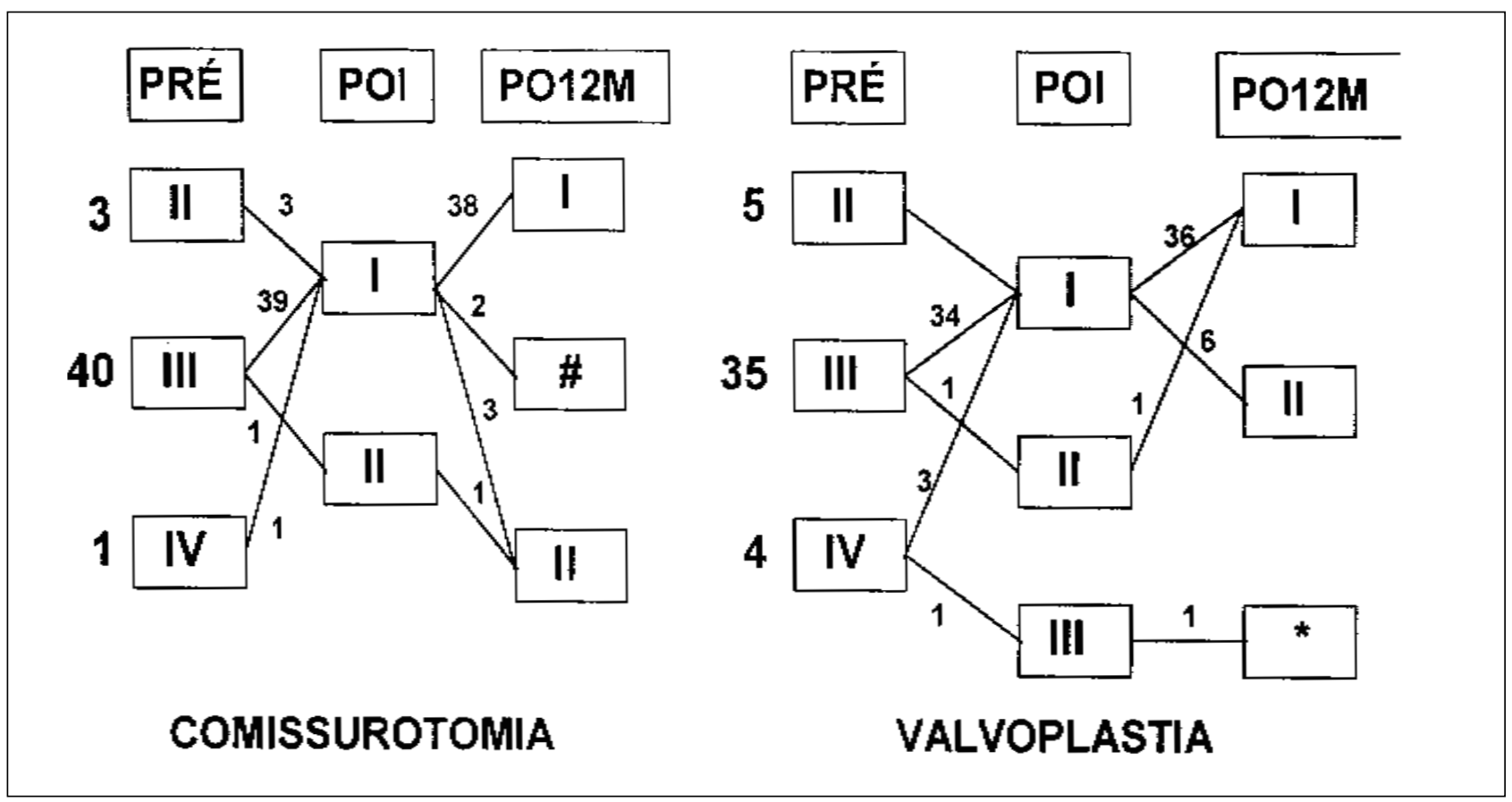

Fig. 1 - Evolução da classe funcional em ambos os grupos nos momentos pré-procedimento (PRÉ), imediatamente após (POI) e após 12 meses de acompanhamento (PO12M).* cirurgia;\#- sem seguimento.

datos ideais para $\mathrm{VMCB}$, visando homogeneizar o material para futura comparação. Acolhemos a existência de correlação inversa entre o referido escore de Wilkins e $\operatorname{col}^{7}$ e o grau de sucesso na $\mathrm{VMCB}{ }^{13}$, pois grandeza inferior a oito associa-se a sucesso imediato superior a $91 \%$ e superior a 9 a $60 \%$ aproximadamente.

A manifestação de CFI/II em todos os pacientes do GC e em 97,7\% (um caso em CF III) dos do GV, no POI, portanto, comportamento idêntico entre os grupos, enquadrou-se no limite superior do verificado na literatura. No PO12M, a CF manteve-se baixa em ambos os grupos, exceção de um $(2,3 \%)$ paciente do GV, que, portador de dupla disfunção mitral pós-VMCB, evoluiu para CF I tão somente após ter sido submetido à substituição da valva mitral.

A aplicação da VMCB associa-se a percentuais de CF I/II semelhantes aos obtidos através da CM. McKay e col ${ }^{14}$ e Arora e col ${ }^{15}$ observaram CF I/II imediatamente pós-procedimento em $87,5 \%$ e $97 \%$ dos casos, respectivamente. Cardoso e col ${ }^{16}$ verificaram $81 \%$ de pacientes submetidos a

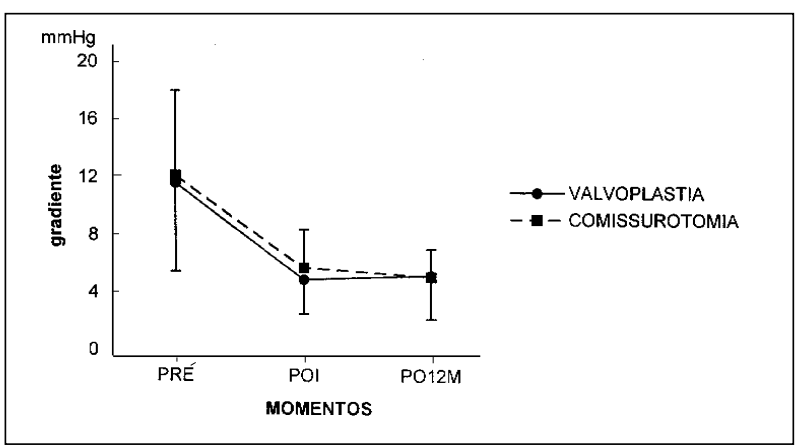

Fig. 2 - Comportamento de média do gradiente transvalvar mitral (mmHg) em ambos os grupos no pré-procedimento (PRÉ), imediatamente após (POI) e após 12 meses de acompanhamento (PO12M).
VMCB em CF I/II no seguimento de um ano.

Análise da AVM é critério importante para avaliação do grau de sucesso da intervenção terapêutica. Por conseguinte, critérios objetivos de avaliação da eficácia devem ser preferencialmente baseados no valor de AVM.

Nosso índice de $100 \%$ de sucesso no GV deve ser creditado, em $1^{\circ}$ à seleção dos pacientes, todos rigidamente considerados como apresentando alta probabilidade de sucesso terapêutico, segundo o escore ecocardiográfico; e, em $2^{\circ}$, ao emprego de técnica altamente padronizada e aplicada por único hemodinamicista. Neste particular, Herrmann e col ${ }^{17}$ obtiveram $96,5 \%$ de sucesso entre 200 pacientes e Cardoso e col ${ }^{16} 91 \%$ em 104 submetidos à VMCB.

Grinberg e col ${ }^{12}$ e Okamura e $\mathrm{col}^{18}$, admitem como fatores negativos de influência no sucesso da abertura valvar por $\mathrm{CM}, \mathrm{e}$, em conseqüência, influenciadores inclusive de aumento na proporção substituição valvar/comissurotomia, a presença de calcificação nos folhetos, comprometimento importante do aparelho subvalvar e associação com IM significativa.

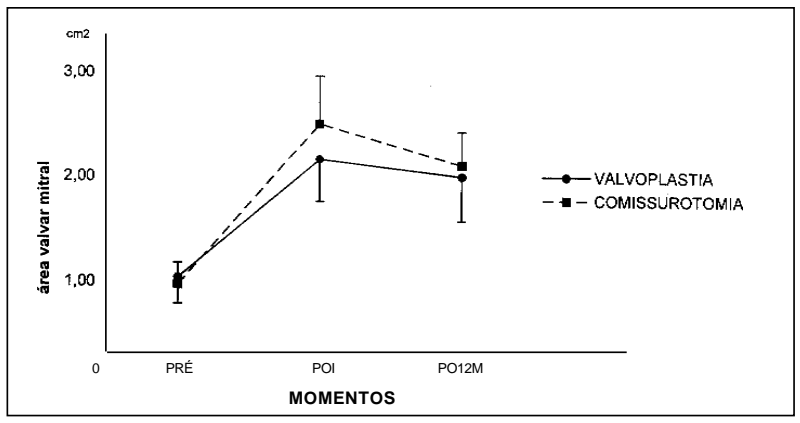

Fig. 3 - Comportamento de média da área valvar mitral $\left(\mathrm{cm}^{2}\right)$ em ambos os grupos no pré-procedimento (PRÉ), imediatamente após (POI) e após 12 meses de acompanhamento (PO12M). 


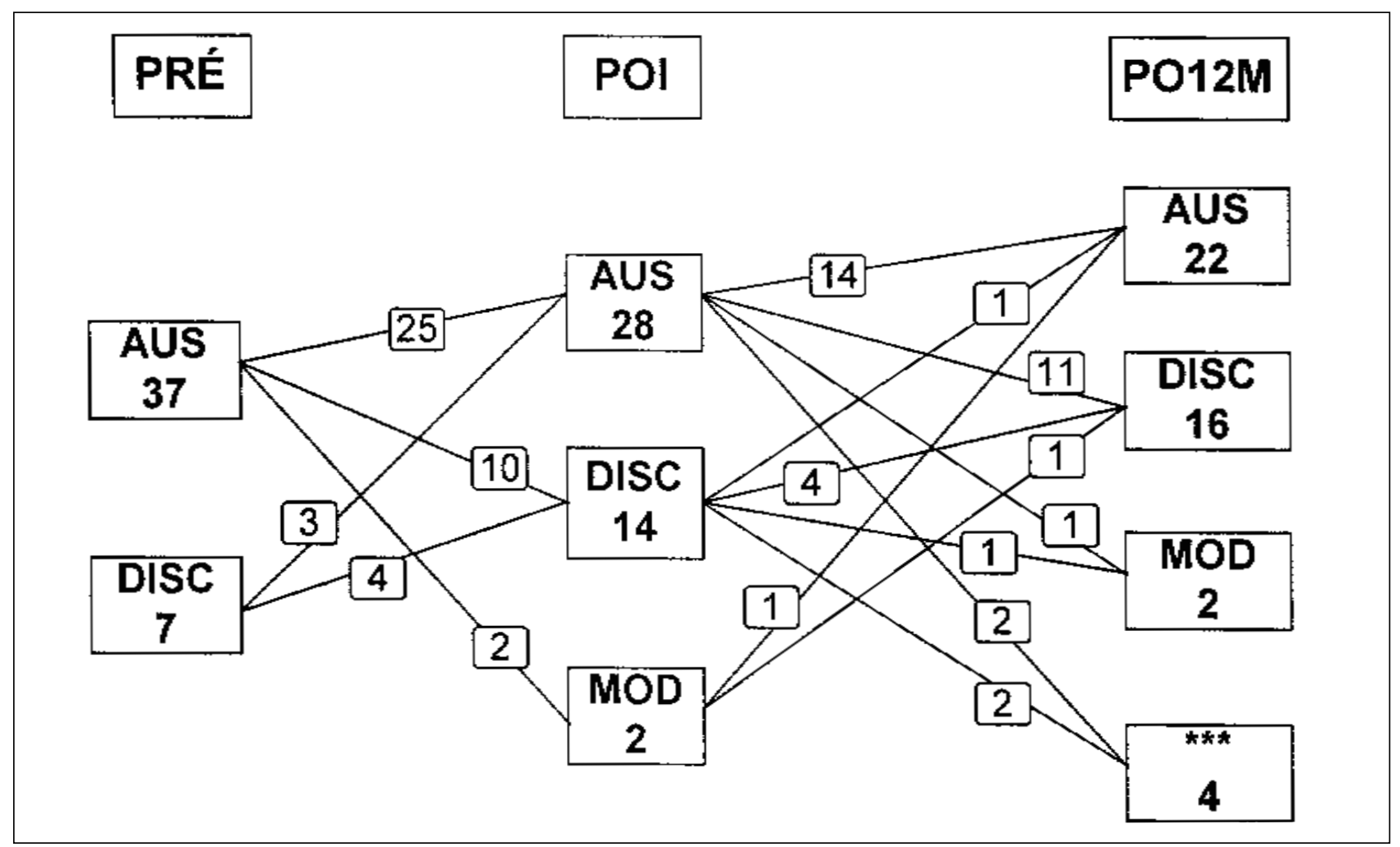

Fig. 4 - Evolução do grau de insuficiência mitral no grupo comissurotomia no pré-procedimento (PRÉ), imediatamente após (POI) e após 12 meses de acompanhamento (PO12M). AUS- ausente; DISC- discreta; MOD- moderada; *** - sem seguimento.

Por outro lado, Block et al ${ }^{19}$ concluíram que escore ecocardiográfico, presença de fibrilação atrial, presença de IM e tamanho do balão utilizado são fatores independentes de influência sobre o grau de aumento da AVM imediatamente pós-VMCB.

Quarenta e um $(93,2 \%)$ dos casos do GV e 36 (81,8\%) dos pacientes do GC encontravam-se em ritmo sinusal no momento PRÉ. A presença de fibrilação atrial deve ser relacionada à evolução mais prolongada da evolução da história natural, tendo sido considerada por Rihal e $\mathrm{col}^{20}$, através de análise univariada e multivariada, fator independente de probabilidade de óbito na evolução pós-CM, ao lado de idade avançada e sexo masculino.

A comparação das médias da AVM no POI em ambos os grupos (tab. II) mostrou ser a CM procedimento efetor de maior grandeza.

Nossos resultados estão em oposição aos encontrados por Reyes e $\mathrm{col}^{21}$ que mostraram AVM pós-VMCB superior à pós-CM, através de estudo hemodinâmico. Acreditamos que esta divergência possa ter ocorrido pela diferença dos métodos na obtenção da AVM.

No PO12M observa-se diminuição significativa da AVM em ambos os grupos (tab. IV e fig. 5). Demonstra-se assim, que nenhum dos dois métodos deixa a valva mitral livre de alguma progressão da doença, provavelmente de natureza cicatricial, sobre os componentes valvar e subvalvar. Decorridos 12 meses, este processo de redução de AVM ocorreu mais notadamente no GC.

Em nosso estudo não observamos no POI através do
ECO, presença de IM importante em qualquer dos dois grupos. Houve maior frequiência de IM moderada no GV do que no GC, dado que é concordante com a literatura. De fato, a VMCB associa-se a IM mais freqüentemente do que a CM. Dezesseis casos do GC e 14 do GV apresentaram IM discreta. Houve aumento de IM no GC em 12 casos sendo de $+\mathrm{em}$ 10 e de $2+$ em dois casos. No GV houve aumento de IM em 13 casos sendo que de + em 10 e de $2+$ em três casos.

Pericardite é complicação pós-operatória e a sua associação com arritmias cardíacas supraventriculares é muito freqüente. Fibrilação e oflutter atrial ocorrem em cerca de 15 a $25 \%$ dos $\operatorname{casos}^{22}$. Em nosso material houve a ocorrência de três casos no POI do GC com pericardite e fibrilação atrial revertidos antes da alta hospitalar sem complicações. Não houve manifestação tardia de recorrência.

A incidência de tamponamento cardíaco pós-VMCB devido a punção transeptal e manipulação de guia e cateteres dentro das cavidades cardíacas ocorre em faixa de 0 a $9,3 \%{ }^{23}$. Nos nossos dois casos de tamponamento cardíaco houve a necessidade apenas de drenagem pericárdica sem complementação com CM.

A realização da VMCB por técnica transeptal provoca comunicação interatrial no POI, detectada por estudo hemodinâmico, ecocardiograma ou cirurgia. Yoshida e $\mathrm{col}^{24}$, valendo-se da ecodopplercardiografia transesofágica com mapeamento de fluxo a cores, demonstraram a presença de comunicação interatrial um dia pós-VMCB em $87,0 \%$ de casos. Observamos ausência de comunicação interatrial no POI, no entanto, a detectamos em um caso no PO12M. Con- 


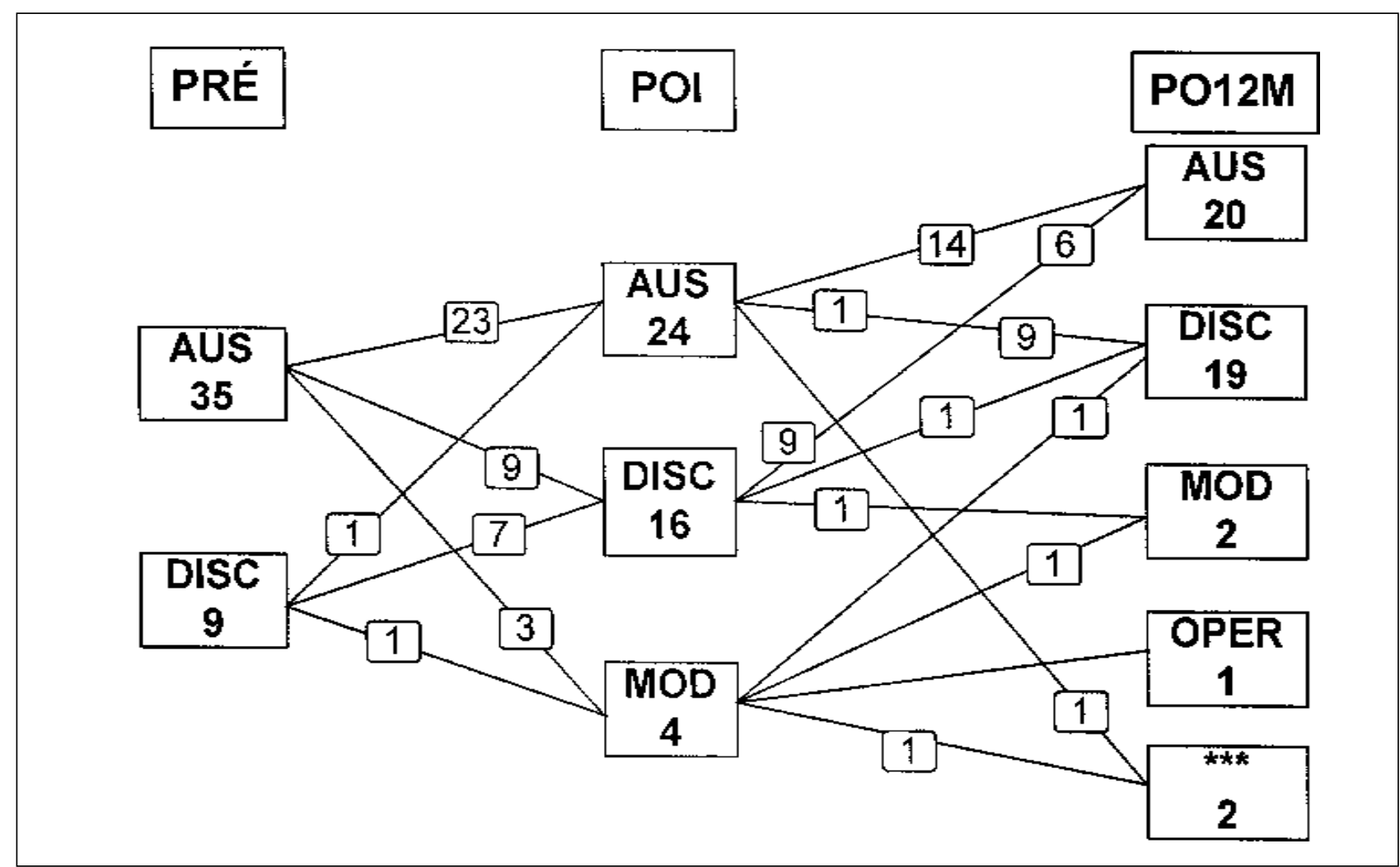

Fig. 5 - Evolução do grau de insuficiência mitral no grupo valvoplastia no pré-procedimento (PRÉ), imediatamente após (POI)e após 12 meses de acompanhamento (PO12M). AUSausente; DISC- discreta; MOD- moderada; *** - sem seguimento; OPER- operado.

tudo, o ecocardiograma transesofágico não foi utilizado e, portanto, seguramente, a presença desta complicação foi subestimada.

O uso de marcapasso temporário no pós-operatório de cirurgia cardíaca visa manter resposta ventricular e débito cardíaco ${ }^{26}$ adequados. Em nossa série, marcapasso epicárdico foi utilizado apenas em um $(2,3 \%)$ caso no GC devido à bradicardia juncional para suporte.

Sangramento em pós-operatório de cirurgia cardíaca em níveis considerados dentro da habitualidade ${ }^{26}$ - cerca de $50 \mathrm{~mL} / \mathrm{h}$ - ocorre em cerca de $70 \%$ dos casos, e sangramento excessivo acontece em menos de $10 \%$ dos casos. Em nosso estudo houve quatro casos de sangramento excessivo no POI do GC, sendo que três $(6,9 \%)$ necessitaram transfusão de sangue e um $(2,3 \%)$ reoperação imediata. Esta baixa incidência deve ser creditada ao fato de se tratar de uma $1^{\text {a }}$ operação.
Uma $(2,3 \%)$ paciente do GV apresentou gravidez ectópica. A melhora da $\mathrm{CF}$ associada ao incremento em AVM, após VMCB ou CM, poderia ser relevante em mulheres com EM em idade fértil, inclusive, podendo-se admitir possibilidade de gestação com menor risco materno-fetal ${ }^{27}$. A presença de um $(2,3 \%)$ caso de fenômeno embólico no GC até o PO12M, em paciente não anticoagulado está de acordo com conhecimento que a embolização sistêmica pode ocorrer em cerca de $25 \%$ de portadores de EM sem anticoagulação ou cirurgia ${ }^{28}$.

Portanto, ambos os métodos foram seguros com baixa taxa de morbidade. Houve uma melhora semelhante no $\mathrm{Ge}$ CF. A AVM apresentou aumento expressivo mais notadamente no GC. Houve redução mais significativa na AVM no GC após 12 meses de acompanhamento, quando comparado com o GV, entretanto, não alterando a classe funcional na maioria dos pacientes em ambos os grupos.

\section{Referências}

1. Meira ZMA, Castilho SRT, Barros MVL - Prevalência da febre reumática em crianças de uma escola da rede pública de Belo Horizonte. Arq Bras Cardiol 1995; 65: $331-4$.

2. Inoue K, Owaki T, Nakamura T, Kitamura F, Miyamoto $\mathrm{N}$-Clinical application of transvenous mitral commissurotomy by a new balloon catheter. J Thorac Cardiovasc Surg 1984; 87: 394-402.

3. Hickey MSJ, Blackstone EH, Kirklin JW, Dean LS - Outcome probabilities and life history after surgical mitral commissurotomy: implications for balloon commissurotomy. J Am Coll Cardiol 1991; 17: 29-42.
4. Herrera JM, Vega JL, Bernal JM, Rabasa JM, Revuelta JM - Open mitral commissurotomy: fourteen to eighteenb year follow-up clinical study. Ann. Thorac. Surg 1993; 55: 641-5.

5. Schmidt-Habelmann P, Civrny J, Meisner H, Park SU, Struck E, Sebening F Open mitral commissurotomy. A new plea for an old operation. J Thorac Cardiovasc Surg 1985; 33: 20-2.

6. New York Heart Association Criteria Committee. Nomenclature and Criteria for Diagnosis of Diseases of the Heart and Great Vessels. $8^{\text {th }}$ ed. Boston: Little Brown, 1979. 
7. Wilkins GT, Weyman AE, Abascal VM, Block PC, Palacios IF - Percutaneous balloon dilatation of the mitral valve: an analysis of echocardiography variables related to outcome and the mechanism of dilatation. Br Heart J 1988; 60: 299 308.

8. Al Zaibag M, Ribeiro PA, Al Kasab S, Al Fagih MR - Percutaneous balloon mitral valvotomy for rheumatic mitral valve stenosis. Lancet 1986; 1: 757-61.

9. Sahn DJ, Demaria A, Kisslo J, Weyman A- The committe in M-mode standardization of the American Society of Echocardiography. Recommendations regarding quatitation in M-mode echocardiography: results of survey of echocardiography measurements. Circulation 1978; 58: 1072-83.

10. Block PC - Who is suitable for percutaneous balloon mitral valvotomy? Int. J. Cardiol 1988; 20: 9-14

11. Horztkotte D, Niehues R, Strauer BE - Pathomorfological aspects, aetiology and natural history of acquires mitral valve stenosis. Eur Heart J 1991;12 (suppl B): 55-60.

12. Grinberg M, Bellotti G, Pileggi F - Aspectos do planejamento terapêutico na estenose mitral. Arq Bras Cardiol 1983; 51: 289-95.

13. Abascal VM, Wilkins GT, Choong CY et al - Echocardiographic evaluation of mitral valve structure and function in patients followed for at least 6 months after percutaneous balloon mitral valvuloplasty. J Am Coll Cardiol 1988; 12: 606-15.

14. Mckay CR, Kawanishi DT, Kotlewski A et al - Improvement in exercise capacity and exercise hemodynamics 3 months after double-balloon, catheter balloon valvuloplasty treatment of patients with symptomatic mitral stenosis. Circulation 1988; 77: 1013-21.

15. Arora R, Mohan N, Kalra GS, Nigan M, Khalilullah M-Immediate and long term results of balloon and surgical closed mitral valvotomy: A randomized comparative study. Am Heart J 1993; 125: 1091-4.

16. Cardoso LF, Rati MAN, Grinberg M et al - Valvoplastia mitral por cateter-balão. Resultados imediatos e seguimento de 1 ano. Arq Bras Cardiol 1992; 58: 445-51.

17. Hermann HC, Ramaswany K, Isner JM et al - Factors influencing immediate results, complications, and short-term follow-up status after Inoue balloon mitral valvotomy. A North American multicenter study. Am Heart J 1992; 124: 160-6.

18. Okamura K, Fukuda I, Maeta H, Mitsui T, Hori M - Two-dimensional echocardiographic evaluation of the severity of mitral stenosis with reference to the prediction for mitral valve commissurotomy or replacement. Clin Cardiol 1986; 9: 99-105.

19. Block PC, Tuzcu EM, Palacios IF - Percutaneous mitral balloon valvotomy. Cardiology Clinics 1991; 9: 271-87.

20. Rihal CS, SchaffHV, Frye RL, Bailey KR, Hammes LN, Holmes DR - Long-term follow-up of patients undergoing closed transventricular mitral commissurotomy: A useful surrogate for percutaneous balloon mitral valvuloplasty? J Am Coll Cardiol 1992; 20: 781-6.

21. Reyes VP, Raj BS, Wynne J et al - Percutaneous balloon valvuloplasty compared with open surgical commissurotomy for mitral stenosis. N Eng J Med 1994; 331: 961-7.

22. Parker JR. FB, Greiner-Hayes C, Bove EL, Mawasti MA, Johnson LW, Eich RH Supraventricular arrhythmias following coronary artery bypass: The effect of preoperative digitalis. J Thorac Cardiovasc Surg 1983; 86: 594-600.

23. Stefanadis C, Toutouza P - Percutaneous mitral valvuloplasty: a decade of experience. Eur Heart J 1995; 16: 1463-8.

24. Yoshida K, Yoshikawa J, Akasaka T et al - Assessment of left-to-right atrial shunting after percutaneous mitral valuloplasty by transesophageal color doppler flow-mapping. Circulation 1989; 80: 1521-6.

25. Hartzler GO, Maloney JD, Curtis JJ, Barnhorst DA - Hemodynamic benefits of atrioventricular sequential pacing after cardiac surgery. Am J Cardiol 1977; 40: 232-42.

26. Starek PJK - In Heart Valve Replacement and Reconstruction: Clinical Issues and Trends. Chicago: Year Book Medical Publishers, 1987.

27. Avila WS, Grinberg M, Decourt LV, Bellotti G-Evolução clínica de portadoras de estenose mitral no ciclo gravídico-puerperal. Arq Bras Cardiol 1992; 58: 359-64.

28. Kellogg F, Liu CK, Fishman IW, Larson R - Systemic and pulmonary emboli before and after mitral commissurotomy. Circulation 1961; 24: 263-6. 